Artículo

\title{
Aptitud actual bajo escenarios de cambio climático para tres cultivos en México
}

\author{
Jesús D. Gómez Díaz \\ Raquel Flores Velázquez \\ Alejandro I. Monterroso Rivas ${ }^{\S}$ \\ Departamento de Suelos-Universidad Autónoma Chapingo. Carretera México-Texcoco km 38.5, Chapingo, \\ Estado de México. CP. 56230. (dgomez@correo.chapingo.mx; raquelflores2088@gmail.com). \\ §Autor para correspondencia: aimrivas@ correo.chapingo.mx.
}

\section{Resumen}

Para la superficie de las tierras agrícolas de México se determinaron las áreas con diferentes grados de aptitud para maíz, sorgo y trigo, todos bajo temporal, para las condiciones actuales y con escenarios de cambio climático estimados con los modelos GFDL, HAGDEM y REA; para los $\mathrm{RCP}$ de 4.5 y $8.5 \mathrm{~W} \mathrm{~m}^{-2}$. La proporción de las tierras agrícolas con algún grado de aptitud para las condiciones actuales es la siguiente: maíz $81.59 \%$, sorgo $61.54 \%$ y trigo $26.95 \%$. De las variables climáticas que determinan la falta de aptitud en el maíz y el trigo en las áreas agrícolas es principalmente la temperatura media anual sola o en combinación con la precipitación, en el sorgo es principalmente la precipitación media anual sola o en combinación con la temperatura media anual. Para los escenarios de cambio climático, con excepción del sorgo, los otros dos cultivos disminuyen de forma considerable la proporción de la superficie de las tierras agrícolas con algún grado de aptitud para estos. El incremento de la temperatura media anual a valores mayores del umbral en que se desarrollan los cultivos de maíz y trigo es la principal causa de perdida de aptitud. La determinación del cambio del grado de aptitud asociada al cambio climático puede usarse para desarrollar políticas públicas para atender la problemática de disminución o perdida de la capacidad de desarrollo de los cultivos.

Palabras claves: adaptación, capacidad productiva, mitigación, vulnerabilidad, zonificación agroecológica.

Recibido: enero de 2020

Aceptado: abril de 2020 


\section{Introducción}

Varios estudios han demostrado que el cambio climático afectará diferencialmente a los países, con importantes vulnerabilidades en regiones de baja latitud (Smith, 2012). México es uno de los países más vulnerables con respecto al cambio climático, dentro de los sectores con mayor afectación posible está la agricultura, esto asociado a que una gran proporción de la actividad se desarrolla bajo condiciones de temporal (82\%) y solo $18 \%$ es de riego (Tinoco et al., 2010).

Al incrementarse la variabilidad climática se estima que aumentará la temperatura y se tendrá la disminución de la humedad en el suelo y con ello se verá comprometida la producción de bienes de consumo por esta actividad, disminuyendo los ingresos económicos de los que depende la población implicada en las actividades agrícolas.

También se estima que se tendrá una disminución en el contenido del carbono orgánico del suelo y con ello una baja en la capacidad productiva de los sistemas agrícolas al afectarse negativamente las propiedades físicas, químicas y biológicas de los suelos (Gómez et al., 2019). Asimismo, las variaciones climáticas traen consigo otras consecuencias negativas para los cultivos, puesto que las plagas, enfermedades y malas hierbas proliferan (Monterroso-Rivas et al., 2018).

Lo anterior, orilla a los agricultores e implicados en el sector a realizar inversiones más altas para adaptar los cultivos a las nuevas condiciones climáticas manifestadas, para poder hacer frente a los cambios deben modificar los patrones de cultivos, uso de insumos, nivel de producción, entre otras acciones (IFPRI, 2009). Sin embargo, la mayoría de los agricultores no cuenta con los ingresos suficientes para hacer frente a las nuevas necesidades.

Así, mientras los países desarrollados han encontrado un equilibrio entre rendimiento y sustentabilidad, los países en desarrollo recurren a métodos menos equilibrados, deteriorando sus recursos naturales al modificar los ecosistemas. Por tanto, el reto consiste en satisfacer la demanda de alimentos a la vez que se mantiene un manejo sustentable de los recursos naturales e incluir acciones de mitigación y manejo sustentable de las tierras agrícolas (Landeros y Pérez, 2009).

Es necesario generar estudios que obtengan información para establecer medidas adaptativas antes que los eventos desfavorables presenten complicación y con ello reducir los impactos negativos, minimizar los problemas socioeconómicos relacionados con el hambre, la reducción de la pobreza, así como generación de alertas a los desastres naturales (FAO, 2018). El objetivo fue analizar la aptitud actual y con cambio climático para tres cultivos en México a nivel regional y así contar con elementos técnicos para recomendar acciones de adaptación en el sector agrícola nacional.

\section{Materiales y métodos}

El proceso se dividió en tres etapas: primero se elaboraron las matrices de requerimientos ambientales para los cultivos del estudio, después para todas las áreas agrícolas del país se elaboraron archivos en formato shapefile con bases de datos de las variables climáticas y se definieron las clases de aptitud actual. Finalmente se aplicaron las proyecciones de cambio climático y se estimaron los cambios en los grados de aptitud de asociados al cambio climático en las tierras agrícolas, mejor descritos a continuación. 


\section{Matrices de requerimientos de los cultivos}

Se elaboraron las matrices de requerimientos ambientales de los tres cultivos del estudio, maíz, sorgo trigo, a partir de revisión bibliográfica en fichas técnicas, guías de FAO y artículos relacionados a los cultivos, dando énfasis a las variables climáticas como son la cantidad y distribución de la precipitación media anual, balances hídricos, temperatura media mensual y anual, además de las condiciones de suelo y de paisaje en el que tienen mejor desarrollo, considerando las áreas en las que actualmente se establecen dichos cultivos. Se usaron cuatro clases de grado de aptitud: apto, moderadamente apto, marginalmente apto y no apto.

\section{Elaboración de los mapas de grado de aptitud de los cultivos}

A partir de las matrices de requerimientos ambientales se elaboraron los mapas de aptitud actual para cada uno de los cultivos del estudio en las áreas con uso agrícola del país, las cuales se delimitaron a partir del mapa de la serie VI del uso del suelo y vegetación (INEGI, 2017), esto con el Software ArcGis 10.3. Para todas las áreas agrícolas del país, se elaboraron archivos en formato shapefile con bases de datos de las variables climáticas como precipitación media anual, temperatura media anual, duración del periodo de crecimiento por humedad disponible, temperatura media del mes más cálido, temperatura media del mes más frio, textura del suelo, pendiente del terreno, condición de drenaje, $\mathrm{pH}$, salinidad y sodicidad así como de la profundidad del suelo.

Se generaron áreas agrícolas con atributos climáticos y de suelos similares con la sobreposición de los valores de los parámetros señalados. Para cada polígono con valores de las variables climáticas y de suelos, se les incluyeron los rangos de las variables climáticas y del suelo para cada cultivo que definen cada clase de aptitud y para cada uno se generó su etiqueta de aptitud actual.

Para este trabajo en la estimación del grado de aptitud bajo escenarios de cambio climático se decidió incluir solo precipitación media anual (PMA) y temperatura media anual (TMA), considerando precipitaciones y temperaturas medias mensuales. La clase de aptitud se definió por los parámetros climáticos más limitantes bajo la siguiente combinación descrita en el Cuadro 1.

Una vez definidas las clases de aptitud, con el Software ArcGis 10.3 se generaron las tablas dinámicas en donde se detalla la superficie para cada combinación de los parámetros que definen el grado de aptitud y se elaboraron los mapas a escala regional (1:250 000) para cada cultivo.

Cuadro 1. Combinación de parámetros climáticos que determinan la clase de aptitud de la tierra para un cultivo particular.

\begin{tabular}{ll}
\hline Parámetros que determinan la aptitud & Criterio \\
\hline Precipitación y temperatura & PMA y TMA mismo nivel de aptitud \\
Precipitación & PMA en un nivel de aptitud menor que TMA \\
Temperatura & TMA en un nivel de aptitud menor que PMA \\
Sin limitaciones & PMA y TMA sin restricciones de aptitud, todas en clase \\
& apta
\end{tabular}

PMA= precipitación media anual; TMA= temperatura media anual. 


\section{Escenarios de cambio climático}

Se aplicaron los escenarios de cambio climático obtenidos de Fernández et al. (2015) para tres modelos: GFDL_CM3, HadGEM2-ES y REA; para el horizonte de tiempo lejano (2070-2099), además de dos RCP: 4.5 y $8.5 \mathrm{~W} \mathrm{~m}^{-2}$. Los modelos se tomaron de la página del Centro de Ciencias de la Atmósfera de la UNAM (UNIATMOS, 2018). Se obtuvieron las razones de cambio para las precipitaciones medias mensuales y las temperaturas medias mensuales para cada área agrícola del país y se calcularon la precipitación media anual y la temperatura media anual a partir de los valores mensuales para cada uno de los escenarios de cambio climático.

Se aplicó el procedimiento descrito en las condiciones actuales para definir las clases de grado de aptitud de las tierras agrícolas para cada cultivo, obteniéndose las bases de datos y sus respectivos mapas para cada cultivo, esto es, con cada modelo se generaron dos tablas dinámicas con las bases de datos y sus mapas, una para el RCP de 4.5 y la otra para el RCP 8.5. Con esta información se analizaron los cambios de aptitud actual en cada escenario de cambio climático para cada cultivo.

\section{Resultados}

\section{Maíz de grano (Zea mays L.) condiciones actuales}

De las 32429966 ha delimitadas como de uso agrícola (INEGI, 2017), 81.59\% tienen algún grado de aptitud para el maíz bajo condiciones de temporal (Cuadro 2), de los cuales $10.91 \%$ no tiene limitantes (apta), 35.67\% como moderadamente aptas y 35\% como marginalmente aptas. Los parámetros climáticos que representan restricciones para este cultivo y que definen las áreas agrícolas como no aptas, están en primer lugar la temperatura.

Cuadro 2. Porcentaje de las tierras agrícolas por aptitud para el cultivo de maíz de temporal bajo condiciones climáticas actuales.

\begin{tabular}{ccccc}
\hline \multirow{2}{*}{$\begin{array}{c}\text { Parámetros que determinan la } \\
\text { aptitud }\end{array}$} & \multicolumn{3}{c}{ Superficie en porcentaje por grado de aptitud actual } \\
\cline { 2 - 5 } & Apto & $\begin{array}{c}\text { Moderadamente } \\
\text { apto }\end{array}$ & $\begin{array}{c}\text { Marginalmente } \\
\text { apto }\end{array}$ & $\begin{array}{c}\text { No } \\
\text { apto }\end{array}$ \\
\hline Precipitación y temperatura & 10.91 & 5.68 & 5.23 & 1.26 \\
Precipitación & & 16.17 & 7.92 & 7.59 \\
Temperatura & & 13.83 & 21.85 & 9.56 \\
Total & 10.91 & 35.67 & 35 & 18.41 \\
\hline
\end{tabular}

Como limitante única o en combinación con otras variables con $10.82 \%$ del total de las tierras agrícolas, la mayor parte de estas áreas presentan temperaturas medias anuales menores a $12{ }^{\circ} \mathrm{C} \mathrm{y}$ se ubican en las zonas altas del Eje Transversal Mexicano y áreas altas de la Sierra Madre Occidental. La precipitación como limitante para que las tierras agrícolas tengan aptitud para este cultivo bajo condiciones de temporal, ya sea sola o en combinación con otras variables representan $8.85 \%$ del total de las tierras agrícolas. 
Estas áreas se asocian a zonas agrícolas con precipitaciones menores a los $350 \mathrm{~mm}$ anuales y algunas áreas con precipitaciones medias anuales mayores a los $4000 \mathrm{~mm}$. Dentro de las áreas agrícolas con precipitaciones medias anuales menores a $350 \mathrm{~mm}$ corresponden en su mayor proporción a zonas bajo riego, aunque hay algunas zonas agrícolas de temporal que tienen esta condición de bajas precipitaciones en donde además de otros cultivos también se siembra maíz, pero con alto grado de siniestralidad.

\section{Escenarios de cambio climático}

En el Cuadro 3 se muestran los resultados del grado de aptitud de las tierras agrícolas del país para maíz de temporal bajo los escenarios de cambio climático para un RCP de 4.5 y 8.5 para el horizonte de tiempo lejano (2 070 a 2 099) y aplicando las razones de cambio para los tres modelos generales de circulación atmosférica (MGCA).

Cuadro 3. Grado de aptitud de las tierras agrícolas de México para maíz bajo escenarios de cambio climático estimados con los modelos GFDL, HADGEM y REA para un RCP de 4.5 y 8.5 .

\begin{tabular}{|c|c|c|c|c|c|c|c|c|c|c|c|}
\hline \multicolumn{3}{|c|}{ Apto } & \multicolumn{3}{|c|}{ Moderadamente apto } & \multicolumn{3}{|c|}{ Marginalmente apto } & \multicolumn{3}{|c|}{ No apto } \\
\hline GFDL & HADGEN & $\overline{\text { REA }}$ & $\overline{\text { GFDL }}$ & ADGEM & REA & GFDL & ADGEM & REA & GFDL & IADGEM & REA \\
\hline \multicolumn{12}{|c|}{ Superficie en porcentaje por grado de aptitud para un RCP de $4.5 \mathrm{~W} \mathrm{~m}^{-2}$} \\
\hline 11.52 & 10.03 & 8.91 & 1.18 & 2.35 & 2.89 & 2.75 & 4.94 & 7.88 & 6.86 & 9.16 & 5.64 \\
\hline- & - & - & 17.63 & 9.38 & 10.75 & 8.19 & 9.63 & 9.42 & 7.16 & 16.23 & 15.9 \\
\hline- & - & - & 7.98 & 5.89 & 6.07 & 11.97 & 11.59 & 14.58 & 24.76 & 20.8 & 17.96 \\
\hline 11.52 & 10.03 & 8.91 & 26.79 & 17.62 & 19.71 & 22.91 & 26.16 & 31.88 & 38.78 & 46.19 & 39.5 \\
\hline \multicolumn{12}{|c|}{ Superficie en porcentaje por grado de aptitud para un $\mathrm{RCP}$ de $8.5 \mathrm{~W} \mathrm{~m}^{-2}$} \\
\hline \multirow[t]{3}{*}{10.79} & 12.14 & 9.13 & 2.51 & 1.08 & 3.03 & 4.45 & 1.03 & 7.09 & 7.64 & 7.62 & 5.73 \\
\hline & & & 14.96 & 14.61 & 11.29 & 12.38 & 9.95 & 9.5 & 6.09 & 9.1 & 14.7 \\
\hline & & & 7.42 & 7.42 & 6.29 & 10.81 & 14.43 & 14.9 & 22.96 & 22.62 & 18.34 \\
\hline 10.79 & 12.14 & 9.13 & 24.89 & 23.11 & 20.61 & 27.65 & 25.41 & 31.49 & 36.68 & 39.34 & 38.77 \\
\hline
\end{tabular}

$\mathrm{P}=$ precipitación media anual; $\mathrm{T}=$ temperatura media anual

En ambos RCP es considerable la disminución de la superficie con algún grado de aptitud para este cultivo, al pasar de $81.59 \%$ del total de las tierras agrícolas a $61.22 \%, 53.81 \%$ y $60.5 \%$ en el RCP 4.5 y a $63.32 \%, 60.66 \%$ y $61.23 \%$ en el RCP 8.5 , para los modelos GFDL, HADGEM y REA, respectivamente. Como se aprecia no hay grandes cambios en los dos RCP, incluso es ligeramente mayor la superficie con algún grado de aptitud en el RCP 8.5 asociado a que se pronostica un menor decremento en la precipitación que en el RCP 4.5.

El incremento de la temperatura estimada en los tres modelos es el factor dominante en la exclusión de las áreas agrícolas para este cultivo, en donde se estima que la temperatura media anual en áreas con algún grado de aptitud bajo condiciones actuales estará con valores superiores a los $26^{\circ} \mathrm{C}$ que es el límite para considerar algún grado de aptitud, la proporción de las áreas agrícolas sin aptitud para este cultivo asociada con la temperatura sola o en combinación. 
Con otras variables como es la precipitación menor a $350 \mathrm{~mm}$ anuales, cambia de $10.82 \%$ a $31.62 \%, 29.96 \%$ y $23.6 \%$ para el RCP 4.5 y $30.6 \%$, 30.24\% y $24.07 \%$ para el RCP 8.5 en los modelos GFDL, HADGEM y REA, respectivamente. Es importante señalar que, aunque algunas áreas agrícolas del país que en condiciones actuales tienen temperaturas medias anuales menores a $12{ }^{\circ} \mathrm{C}$ y que en estos escenarios su temperatura será mayor a este umbral por lo que presentarán algún grado de aptitud para este cultivo.

Sin embargo, la superficie que incrementa su temperatura media anual a valores superiores de 26 ${ }^{\circ} \mathrm{C}$ es considerablemente mayor. Como se aprecia, la proporción de la superficie que pasa a no apta es similar en los dos RCPs en cada modelo. La proporción de las áreas agrícolas del país que no tendrán aptitud para este cultivo bajo condiciones de temporal derivadas principalmente de la disminución de la precipitación a valores menores a $350 \mathrm{~mm}$ anuales pasan de $8.85 \%$ a $14.02 \%$, $25.39 \%$ y $21.54 \%$ en el RCP 4.5 y de $12.05 \%, 14.96 \%$ y $18.69 \%$ en el RCP 8.5 , para los modelos GFDL, HADGEM y REA, respectivamente. En los tres modelos en el RCP 8.5 se tiene una menor afectación en la superficie con algún grado de aptitud, asociado a que se estima que la disminución de la precipitación en diferentes regiones de México será menos severa que para el RCP 4.5.

\section{Sorgo de grano (Sorghum bicolor L.) condiciones actuales}

Del total de las tierras agrícolas, $61.54 \%$ tienen algún grado de aptitud para sorgo de grano bajo condiciones de temporal (Cuadro 4). Los parámetros climáticos que representan restricciones para este cultivo y que definen las áreas agrícolas como no aptas, están en primer lugar la precipitación como limitante única o en combinación con otras variables, $28.16 \%$ del total de las tierras agrícolas, estas áreas presentan precipitación media anual menores a $400 \mathrm{~mm}$ o mayores a $1800 \mathrm{~mm}$.

Cuadro 4. Porcentaje de las tierras agrícolas por aptitud para sorgo de grano de temporal bajo condiciones climáticas actuales.

\begin{tabular}{ccccc}
\hline \multirow{2}{*}{$\begin{array}{c}\text { Parámetros que determinan la } \\
\text { aptitud }\end{array}$} & \multicolumn{3}{c}{ Superficie en porcentaje por grado de aptitud actual } \\
\cline { 2 - 5 } & Apto & Moderadamente apto & Marginalmente apto & No apto \\
\hline Precipitación y temperatura & 5.96 & 3.33 & 14.74 & 12.9 \\
Precipitación & & 5.5 & 3.83 & 15.26 \\
Temperatura & & 10.6 & 17.58 & 10.3 \\
Total & 5.96 & 19.43 & 36.15 & 38.46 \\
\hline
\end{tabular}

La temperatura como limitante para que las tierras agrícolas tengan aptitud para este cultivo bajo condiciones de temporal, ya sea sola o en combinación con la precipitación representan 23.20\% del total de las tierras agrícolas, estas áreas se asocian a zonas agrícolas con temperaturas medias anuales menores a $15{ }^{\circ} \mathrm{C}$ o mayores a $28{ }^{\circ} \mathrm{C}$. En algunas regiones del país donde se establece este cultivo bajo riego no necesariamente se cumple la condición de precipitación media anual.

\section{Escenarios de cambio climático}

En el Cuadro 5 se muestran los resultados del grado de aptitud de las tierras agrícolas del país para sorgo de grano en condiciones de temporal para los escenarios de cambio climático para los RCP de 4.5 y 8.5 para el horizonte de tiempo lejano (2 070 a 2099 ) y aplicando las razones de cambio para los tres modelos generales de circulación atmosférica (MGCA) de este estudio. 
Cuadro 5. Grado de aptitud de las tierras agrícolas de México para de sorgo de grano bajo temporal para los escenarios de cambio climático estimados con los modelos GFDL, HADGEM y REA para un RCP de 4.5 y 8.5.

\begin{tabular}{|c|c|c|c|c|c|c|c|c|c|c|c|}
\hline \multicolumn{3}{|c|}{ Apto } & \multicolumn{3}{|c|}{ Moderadamente apto } & \multicolumn{3}{|c|}{ Marginalmente apto } & \multicolumn{3}{|c|}{ No apto } \\
\hline \multicolumn{3}{|c|}{$\begin{array}{l}\text { GFDL HADGEM REA } \\
\text { HFD }\end{array}$} & \multicolumn{3}{|c|}{$\begin{array}{l}\text { GFDL HADGEM REA } \\
\end{array}$} & \multicolumn{3}{|c|}{ GFDL HADGEM REA } & \multicolumn{3}{|c|}{ GFDL HADGEM REA } \\
\hline \multicolumn{12}{|c|}{ Superficie en porcentaje por grado de aptitud para un $\mathrm{RCP}$ de $4.5 \mathrm{~W} \mathrm{~m}^{-2}$} \\
\hline \multirow[t]{3}{*}{6.7} & 6.12 & 5.09 & 2.81 & 4.32 & 2.29 & 11.43 & 12.23 & 10.87 & 12.24 & 16.05 & 16.68 \\
\hline & & & 5.55 & 4.06 & 6.55 & 12.43 & 5.6 & 3.9 & 13.47 & 22.26 & 21.71 \\
\hline & & & 5.84 & 3.61 & 4.43 & 20.82 & 17.12 & 20.85 & 8.71 & 8.63 & 7.63 \\
\hline 6.7 & 6.12 & 5.09 & 14.2 & 11.99 & 13.27 & 44.68 & 34.95 & 35.62 & 34.42 & 46.94 & 46.02 \\
\hline \multicolumn{12}{|c|}{ Superficie en porcentaje por grado de aptitud para un RCP de $8.5 \mathrm{~W} \mathrm{~m}^{-2}$} \\
\hline \multirow[t]{3}{*}{6.46} & 6.31 & 5.29 & 3.79 & 3.37 & 2.16 & 14.05 & 11.11 & 11 & 12.55 & 13.1 & 15.78 \\
\hline & & & 6.27 & 4.82 & 6.56 & 9.55 & 10.25 & 3.76 & 14.63 & 16.28 & 21.09 \\
\hline & & & 4.97 & 6.28 & 4.55 & 18.15 & 20.00 & 21.84 & 9.59 & 8.48 & 7.97 \\
\hline 6.46 & 6.31 & 5.29 & 15.03 & 14.47 & 13.27 & 41.75 & 41.36 & 36.6 & 36.77 & 37.86 & 44.84 \\
\hline
\end{tabular}

En el RCP 4.5 resalta que en el modelo GFDL se incrementará la superficie con algún grado de aptitud para este cultivo al pasar de $61.54 \%$ a $65.58 \%$, en cambio para los modelos HADGEM y REA la superficie con algún grado de aptitud para el desarrollo de este cultivo disminuye a 53.06\% y $53.98 \%$, respectivamente.

Para el RCP 8.5 la proporción de la superficie de las tierras agrícolas con algún grado de aptitud aumenta ligeramente con respecto a las condiciones actuales en los modelos GFDL y HADGEM con $63.23 \%$ y $62.14 \%$, respectivamente y disminuye a $55.16 \%$ en el modelo REA. Con respecto a los resultados del RCP 4.5 el mayor cambio se estima con el modelo HADGEM que se incrementa en $10 \%$ del total de la superficie agrícola, mientras en los otros modelos los cambios son menores.

La proporción de las áreas agrícolas del país que no tendrán aptitud para este cultivo bajo condiciones de temporal derivadas principalmente de la disminución de la precipitación a valores menores a $400 \mathrm{~mm}$ anuales pasan de $28.16 \%$ bajo condiciones actuales a $23.9 \%, 38.31 \%$ y $38.39 \%$ en el RCP 4.5 y de $27.18 \%, 29.38 \%$ y $35.87 \%$ en el RCP 8.5 para los modelos GFDL, HADGEM y REA, respectivamente.

El modelo GFDL en el RCP 4.5 se estima un incremento en la superficie que está en el rango de precipitación con algún grado de aptitud, por el incremento en la superficie con precipitaciones medias anuales menores a $1800 \mathrm{~mm}$, mientras que en el RCP 8.5 el cambio de la superficie dentro del rango de precipitación con algún grado de aptitud es similar a las condiciones actuales.

En el modelo HADGEM para el RCP 4.5 hay una disminución considerable de la superficie con el rango de precipitación con algún grado de aptitud, mientras que en el RCP 8.5 se incrementa la precipitación con respecto al $\mathrm{RCP}$ anterior, principalmente en las áreas que tuvieron valores menores a $400 \mathrm{~mm}$ anuales, para tener una superficie similar a las condiciones actuales. En el modelo REA en ambos RCPs hay una disminución de la superficie con algún grado de aptitud en el rango de precipitación definido para este cultivo. 
El incremento de la temperatura estimada en los tres modelos reporta un incremento en las áreas con temperatura media anual mayor a $15^{\circ} \mathrm{C}$, así como un incremento a las áreas con temperaturas mayores a $28{ }^{\circ} \mathrm{C}$, por lo que la proporción de las áreas agrícolas sin aptitud para este cultivo asociada con la temperatura sola o en combinación con otras variables como es la precipitación menor a $400 \mathrm{~mm}$ anuales o mayor a $1800 \mathrm{~mm}$ anuales, cambia ligeramente de $23.2 \%$ en condiciones actuales a $20.94 \%, 24.68 \%$ y $24.31 \%$ para el RCP 4.5 y $22.14 \%, 21.58 \%$ y $23.75 \%$ para el RCP 8.5, en los modelos GFDL, HADGEM y REA, respectivamente.

En el modelo GFDL se tiene un incremento en la superficie con algún grado de aptitud por este parámetro en los dos RCPs siendo superior en el RCP 4.5. Para el modelo HADGEM se estima una ligera reducción de la superficie con algún grado de aptitud en el RCP 4.5, mientras que se reporta un ligero incremento para el RCP 8.5. Para el modelo REA los valores para ambos RCPs son similares en la proporción de superficie con algún grado de aptitud.

\section{Trigo de grano (Triticum spp. L.) condiciones actuales}

Del total de las tierras agrícolas del país solo $26.95 \%$ tienen algún grado de aptitud para el cultivo de trigo bajo condiciones de temporal (Cuadro 6). Los parámetros climáticos que representan restricciones para este cultivo y que definen las áreas agrícolas como no aptas, están en primer lugar la temperatura media anual mayor a $18{ }^{\circ} \mathrm{C}$ que sola o en combinación con la precipitación representan $71.79 \%$ del total de las tierras agrícolas.

Cuadro 6. Porcentaje de las tierras agrícolas por aptitud para el cultivo de trigo de grano de temporal bajo condiciones climáticas actuales.

\begin{tabular}{ccccc}
\hline \multirow{2}{*}{$\begin{array}{c}\text { Parámetros que determinan la } \\
\text { aptitud }\end{array}$} & \multicolumn{3}{c}{ Superficie en porcentaje por grado de aptitud actual } \\
\cline { 2 - 5 } & Apto & Moderadamente apto & Marginalmente apto & No apto \\
\hline Precipitación y temperatura & 2.61 & 4.17 & 7.68 & 31.08 \\
Precipitación & - & 3.73 & 0.77 & 1.26 \\
Temperatura & - & 3.53 & 4.46 & 40.71 \\
Total & 2.61 & 11.43 & 12.91 & 73.05 \\
\hline
\end{tabular}

La precipitación como factor limitante para que no sean aptas las tierras agrícolas para este cultivo por tener valores mayores a los $2000 \mathrm{~mm}$ anuales o menores a los $300 \mathrm{~mm}$ anuales, sola o en combinación con otros factores representan $32.34 \%$ de la superficie agrícola del país. Cabe señalar, que algunas de las regiones productoras de trigo como son las áreas de la planicie costera del pacifico norte en Sonora, el cultivo se desarrolla en invierno y bajo riego.

\section{Escenarios de cambio climático}

En el Cuadro 7 se muestran los resultados del grado de aptitud de las tierras agrícolas del país para trigo en condiciones de temporal para los escenarios de cambio climático para los RCP de $4.5 \mathrm{y}$ 8.5 para el horizonte de tiempo lejano (2 070 a 2099 ) y aplicando las razones de cambio para los tres modelos generales de circulación atmosférica (MGCA) de este estudio. 
Cuadro 7. Grado de aptitud de las tierras agrícolas de México para el cultivo de trigo de temporal para los escenarios de cambio climático estimados con los modelos GFDL, HADGEM y REA para un RCP de 4.5 y 8.5 .

\begin{tabular}{|c|c|c|c|c|c|c|c|c|c|c|c|}
\hline \multicolumn{3}{|c|}{ Apto } & \multicolumn{3}{|c|}{ Moderadamente apto } & \multicolumn{3}{|c|}{ Marginalmente apto } & \multicolumn{3}{|c|}{ No apto } \\
\hline GFDL & IADGE & REA & GFDL & ADGE & REA & GFDL & ADGE & REA & GFDL & IADGEM & REA \\
\hline \multicolumn{12}{|c|}{ Superficie en porcentaje por grado de aptitud para un RCP de $4.5 \mathrm{~W} \mathrm{~m}^{-2}$} \\
\hline \multirow[t]{3}{*}{2.08} & 1.77 & 1.7 & 2.27 & 2.81 & 2.51 & 3.33 & 4.51 & 4.60 & 39.19 & 45.33 & 43.91 \\
\hline & & & 2.05 & 2.45 & 1.91 & 0.33 & 0.45 & 1.26 & 0.31 & 1.44 & 2.23 \\
\hline & & & 3.13 & 3.31 & 2.64 & 2.42 & 1.95 & 2.02 & 44.91 & 35.98 & 37.23 \\
\hline 2.08 & 1.77 & 1.7 & 7.45 & 8.57 & 7.04 & 6.06 & 6.91 & 7.88 & 84.41 & 82.75 & 83.37 \\
\hline \multicolumn{12}{|c|}{ Superficie en porcentaje por grado de aptitud para un RCP de $8.5 \mathrm{~W} \mathrm{~m}^{-2}$} \\
\hline \multirow[t]{3}{*}{2.25} & 2.28 & 1.69 & 2.45 & 2.89 & 2.52 & 4.09 & 2.91 & 4.66 & 40.57 & 41.16 & 42.83 \\
\hline & & & 2.52 & 1.88 & 1.94 & 0.35 & 0.22 & 1.27 & 1.36 & 0.48 & 2.16 \\
\hline & & & 2.89 & 3.53 & 2.57 & 2.56 & 3.5 & 1.96 & 40.96 & 41.16 & 38.41 \\
\hline 2.25 & 2.28 & 1.69 & 7.86 & 8.3 & 7.04 & 7 & 6.63 & 7.89 & 82.89 & 82.8 & 83.4 \\
\hline
\end{tabular}

En ambos RCP es considerable la disminución de la superficie con algún grado de aptitud para este cultivo, al pasar en condiciones actuales de $26.95 \%$ del total de las tierras agrícolas a $15.59 \%$, $17.25 \%$ y $16.62 \%$ en el RCP 4.5 y a $17.11 \%, 17.21 \%$ y $16.62 \%$ en el RCP 8.5 , para los modelos GFDL, HADGEM y REA, respectivamente. Como se aprecia solo en el modelo GFDL hay cambio entre los dos RCPs, con una menor disminución de la superficie con algún grado de aptitud en el RCP 8.5 comparado con el de 4.5. En los otros dos modelos los resultados son similares para las dos RCPs.

El incremento de la temperatura estimada en los tres modelos es el factor dominante en la exclusión de las áreas agrícolas para este cultivo, en donde se estima que la temperatura media anual en áreas con algún grado de aptitud bajo condiciones actuales, estará con valores superiores a los $18{ }^{\circ} \mathrm{C}$ que es el límite para considerar algún grado de aptitud, la proporción de las áreas agrícolas sin aptitud para este cultivo asociada con la temperatura sola o en combinación con otras variables como es la precipitación menor a $300 \mathrm{~mm}$ anuales, cambia de $71.79 \%$.

En condiciones actuales a $84.1 \%, 81.31 \%$ y $81.15 \%$ para el RCP 4.5 y $81.53 \%, 82.31 \%$ y $81.22 \%$ para el RCP 8.5, en los modelos GFDL, HADGEM y REA, respectivamente. Como se observa, la proporción de la superficie que pasa a no apta es similar en los dos RCPs para los modelos HADGEN y REA, en el modelo GFDL es mayor en el RCP 4.5. La proporción de las áreas agrícolas del país que no tendrán aptitud para este cultivo bajo condiciones de temporal derivadas principalmente de la disminución de la precipitación a valores menores a $300 \mathrm{~mm}$ anuales o incremento.

En la precipitación a más de $2000 \mathrm{~mm}$ anuales pasan de $32.34 \%$ en condiciones actuales a $39.5 \%$, $46.77 \%$ y $46.15 \%$ en el RCP 4.5 y a $41.93 \%$, $41.64 \%$ y $44.98 \%$ en el RCP 8.5 , para los modelos GFDL, HADGEM y REA, respectivamente. En los tres modelos en el RCP 4.5 se tiene una menor afectación en la superficie con algún grado de aptitud. 


\section{Discusión}

Para los tres modelos generales de circulación atmosférica los escenarios de cambio climático estimados contemplan un incremento de la temperatura que varía en las diferentes regiones de México, así como entre cada modelo; sin embargo, las expectativas es que todo el país tenga temperaturas más altas. El calentamiento proyectado puede exacerbar varias vulnerabilidades existentes entre las que se consideran la aptitud de las tierras para diferentes cultivos (Sivakumar et al., 2005; Trenberth et al., 2007; Ojeda et al., 2011).

Las temperaturas más altas pueden tener impactos negativos y algunos positivos en el desarrollo de los cultivos (Childress et al., 2015), prevaleciendo los negativos en este estudio en donde el maíz y trigo muestran una reducción en la superficie con algún grado de aptitud asociado al incremento de la temperatura, mientras que el sorgo se tiene un ligero incremento en la superficie con algún grado de aptitud para el modelo GFDL y ligeros cambios para los otros dos modelos.

Virtualmente cada aspecto del sector agrícola es altamente sensitivo a las condiciones climáticas y como se indicó, los cambios en el clima pueden tener efectos positivos y efectos negativos, dependiendo de la región y los cambios que se tengan. La agricultura en México es vulnerable en algunos de los impactos del cambio climático predichos, incluyendo los incrementos en la temperatura, disminución en la precipitación y menor abastecimiento de agua, así como incrementos en las plagas y enfermedades de los cultivos (Monterroso-Rivas et al., 2018).

Los cambios en la frecuencia y severidad de las sequías, en la cantidad y distribución de la precipitación, las inundaciones, entre otras, también representan nuevos retos para los agricultores (Childress et al., 2015). Se estima que el incremento de las temperaturas, que será más pronunciado en el verano, tendrá como consecuencia mayor frecuencia y severidad de las sequías, reduciendo la humedad disponible (Gornall et al., 2010; Hatfield et al., 2011).

para los cultivos y también en la disponibilidad de agua en las presas y abatimiento en los acuíferos, estos cambios tendrán un impacto significativo en el rendimiento de los cultivos (Hatfield et al., 2011). El incremento en las temperaturas puede hacer que varios cultivos crezcan más rápido, lo que puede hacerlos más vulnerable a la afectación por estrés de humedad en las etapas más sensitivas del cultivo y con ello mayor incertidumbre en los rendimientos (Islam et al., 2012).

Para cualquier cultivo en particular los efectos del incremento en la temperatura dependerán en la temperatura optima en la que se desarrolla cada cultivo, por lo que, si esta fuera del rango en que se da el crecimiento, la polinización y la formación de la semilla, se verá afectado el rendimiento del cultivo. Muchos cultivos muestran efectos del umbral térmico en que pueden desarrollarse, por lo que si el calentamiento supera la temperatura del rango en que el cultivo se desarrolla, los rendimientos pueden disminuir considerablemente o ser cero (Hatfield et al., 2011).

Los cultivos de temporal, especialmente el trigo, muestran reducción de rendimientos bajo varios escenarios de cambio climático debido al aumento en la temperatura y al estrés hídrico (Ko et al., 2012). Asimismo, los cultivos bajo riego como el maíz son sensibles al incremento de la temperatura aun cuando se les aplican los riegos que se requieren (Islam et al., 2012). El aumento en la temperatura incrementa la demanda de agua para muchos cultivos. 
Independientemente de los cambios en la precipitación, las temperaturas más cálidas durante la temporada de crecimiento aumentarán la demanda de evaporación, lo que aumenta las tasas de evapotranspiración y con ello la demanda de agua de los cultivos (Udall, 2013). Ante este panorama, el sector agrícola es potencialmente vulnerable al cambio climático y a la disminución o perdida de las cosechas (Childress et al., 2015), por lo que se hacen necesarios estudios que estimen la afectación de estos ante los escenarios de cambio climático.

\section{Conclusiones}

El grado de aptitud para las condiciones actuales varía considerablemente entre los cultivos, el maíz es el que presenta mayor proporción de las tierras agrícolas con algún grado de aptitud con $81.59 \%$, seguido del sorgo con $61.54 \%$ y con proporción menor están el trigo que tiene $26.95 \%$. De las variables climáticas que determinan la falta de aptitud en el maíz y el trigo en las áreas agrícolas es principalmente la temperatura media anual sola o en combinación con la precipitación, el sorgo es principalmente la precipitación media anual sola o en combinación con la temperatura media anual.

Particularmente para el maíz se incrementa considerablemente la superficie que en condiciones actuales no es apta por temperaturas medias anuales con valores menores al límite inferior en que se desarrolla el cultivo y que en los escenarios de cambio climático se incrementará y tendrán aptitud estas áreas, pero es considerablemente mayor la superficie que deja de tener aptitud al incrementarse la temperatura a valores superiores del límite superior que se desarrolla este cultivo.

Se determinó la afectación en el grado de aptitud de las tierras agrícolas para los cultivos considerados y se tiene la ubicación geográfica de estas afectaciones, mismos que pueden usarse para desarrollar políticas públicas para atender la problemática de disminución o perdida de la capacidad de desarrollo de estos cultivos, implementando programas de mitigación y adaptación a las condiciones que se pueden presentan bajo los escenarios de cambio climático y dar alternativas a los productores de las áreas con afectaciones.

\section{Literatura citada}

Childress, A.; Kelly, E. and Travis, W. 2015. Chapter 6 -agriculture sector. In: Colorado Climate Change Vulnerability Study, edited by Eric Gordon and Dennis Ojima. University of Colorado, Boulder, CO and Colorado State University, Fort Collins, CO. 75-88 pp.

FAO. 2018. Cambio climático. Retrieved from http://www.fao.org/climate-change/es/.

Fernández, E. A.; Zavala, H. J.; Romero, C. R.; Conde, A. C. y Trejo, V. I. 2015. Actualización de los escenarios de cambio climático para estudios de impactos, vulnerabilidad y adaptación. Ciudad de México, México. <http://atlasclimatico.unam.mx/AECC/servmapas/>.

Gómez-Díaz, J.; Monterroso, A.; Ruiz, P.; Lechuga, L.; Álvarez, A. and Asensio, C. 2019. "Soil moisture regimes in Mexico in a global $1.5^{\circ} \mathrm{C}$ warming scenario". International Journal of Climate Change Strategies and Management. 4(11):465-482. https://doi.org/10.1108/ IJCCSM-08-2018-0062.

Gornall, J.; Betts, R.; Burke, E.; Clark, R.; Camp, J.; Willet, K. and Wiltshire, A. 2010. Implications of climate change for agricultural productivity in the early twenty-first century. Phil. Trans. R. Soc. B. 365:2973-2989. 
Hatfield, J. L.; Boote, K. J.; Kimball, B. A.; Ziska, L. H.; Izaurralde, R. C.; Ort, D.; Thomson, A. M. and Wolfe, D. 2011. Climate impacts on agriculture: implications for crop production. Agron. J. 103(2):351-370.

IFPRI. 2009. Cambio Climático: El impacto en la agricultura y los costos de adaptación, Washington, DC. 1-19 pp.

INEGI. 2017. Datos vectoriales escala 1:250 000 serie VI (Capa Unión) del Uso de Suelo y Vegetación. Instituto Nacional de Estadística y Geografía. México.

Islam, A.; Ahuja, L.R.; Garcia, L.A.; Ma, L.; Saseendran, A.S. and Trout, T. J. 2012. Modeling the impacts of climate change on irrigated corn production in the Central Great Plains. Agricultural Water Management. 110(1):94-108.

Ko, J.; Ahuja, L.; Saseendran, S.; Green, T.; Ma, L.; Nielsen, D. and Walthall, C. 2012. Climate change impacts on dryland cropping systems in the Central Great Plains, USA. Climatic Change. 111: 445-472.

Landeros S. C. y Pérez V. A. 2009. Agricultura y deterioro ambiental. Elementos. 73:19-25.

Medina, G. G.; Ruiz, C. J. A. y Bravo, L. A. G. 2006. Definición y clasificación de la sequía. In: sequía: vulnerabilidad, impacto y tecnología para afrontarla en el Norte Centro de México. (Comp.). Bravo, L. A.; Salinas, G. H. y Rumayor, R. A. INIFAP, CIRNOC, CEZAC. Calera de V. R., Zacatecas. Libro técnico núm. 4. $2^{\text {da }}$ (Ed). México. 300 p.

Monterroso-Rivas, A. I.; Conde-Alvarez, A. C.; Perez-Damian, J. L.; Lopez-Blanco, J.; GaytanDimaz, M. and Gómez-Diaz, J. D. 2018. Multi-temporal assessment of vulnerability to climate change: insights from the agricultural sector in Mexico. Climate Change. 147:457-473.

Ojeda, B. W.; Sifuentes, I. E.; Íñiguez, C. M. y Montero, M. M. J. 2011. Impacto del cambio climático en el desarrollo y requerimientos hídricos de los cultivos. Agrociencia. 45:1-11.

Sivakumar, M. V. K.; Das, H. P. and Brunini, O. 2005. Impacts of present and future climate variability and change on agriculture and forestry in the arid and semi-arid tropics. In: increasing climate variability and change. Salinger, J. M.; Sivakumar, V. K. and Motha, R. P. (Eds.). Springer, USA. 31-72 pp.

Smith, P. 2012. "Soils and climate change". Curr. Opin. Environ. Sust. 4(5):539-544.

Tinoco-Rueda, J. A.; Gómez-Díaz, J. D. y Monterroso-Rivas, A. I. 2010. Efectos del cambio climático en la distribución potencial del maíz en el estado de Jalisco, México. Terra Latinoamericana. 2(29):161-168.

Trenberth, K. E.; Jones, P. D.; Ambenje, P.; Bojariu, R.; Easterling, D. R.; Kleint-Tank, A.; Parker, D.; Rahimzadeh, F.; Renwick, J. A.; Rusticucci, M.; Soden, B. and Zhai, P. 2007. Observations: surface and atmospheric climate change. Climate Change 2007: the physical science basis. Contribution of wg $i$ to the fourth assessment report of the IPCC. Cambridge University Press. 321 p.

Udall, B. (2013). "Water: Impacts, Risks, and Adaptation." In: Assessment of Climate Change in the Southwest United States: A Report Prepared for the National Climate Assessment, edited by G. Garfin; A. Jardine; R. Merideth; M. Black and S. LeRoy, 197-217. A report by the Southwest Climate Alliance. Washington, DC: Island Press.

UNIATMOS. 2018. Atlas climático digital de México. Centro de Ciencias de la Atmosfera, Universidad Nacional Autónoma de México (UNAM). 